\title{
Zur Entwicklung des Technologietransfers für KMU in den neuen Bundesländern - konzeptionelle Überlegungen, Probleme und Anforderungen
}

\author{
Klaus Däumichen
}

\section{Entwicklung neuer Technologien und deren schnelle Umsetzung/Technologie- transfer und Innovationsmanagement}

Innovation ist in den letzten Jahren zu einem Schlüsselbegriff wirtschaftlichen Handelns geworden. Phänomene wie Verkürzung der Produktlebenszyklen, technologischer Wandel und Öffnung der nationalen Märkte kennzeichnen die Rahmenbedingungen, unter denen das Prinzip der permanenten Innovation nicht nur zum entscheidenden Kriterium der Wettbewersfähigkeit des einzelnen Unternehmens wird, sondern darüber hinaus zum Hauptfaktor der Überlebensfähigkeit nationaler Volkswirtschaften. Einer Studie des Fraunhofer Instituts für Innovationsforschung zufolge beschäftigen z. B. High-Tech-Unternehmen in den neuen Bundesländern nach fünf Jahren durchschnittlich 15 Angestellte. In den übrigen Industriebranchen liegt diese Zahl rund zwei Drittel niedriger.

Die wissenschaftliche Diskussion des Begriffes „Innovation“ wird an Hand sehr unterschiedlicher Ansätze und unter Rückgriff auf in anderen Zusammenhängen entwickelte Theorien geführt. Seit Mitte der siebziger Jahre konzentriert sich die wissenschaftliche Auseinandersetzung vor allem auf die Fragen der Initiierung, Steuerung und Durchfuihrung von Innovationsprozessen in Unternehmen. Innovationsmanagement ist der zusammenfassende Begriff, unter dem die Frage nach der Steuerung und Gestaltung von Innovationsprozessen ihren Einzug in die Management- und Organisationstheorie und -praxis gefunden hat.

Auf der theoretischen Ebene sowie zur betrieblichen Praxis des Innovationsmanagementes liegen die unterschiedlichsten Untersuchungen vor. Es fehlt aber eine fundierte Transformation der theoretischen Erkenntnisse des Innovationsmanagementes auf die Ebene der betrieblichen Praxis. Diesbezügliche Fragestellungen sind ${ }^{1}$ :

- Welche Schritte sind notwendig, um eine fundierte Transformation der theoretischen Erkenntnisse über das Innovationsmanagement auf die Ebene des praktischen Handelns - unter Berücksichtigung der Erfordernisse der KMU - zu vollziehen?

- Wie kann das Innovationsmanagement in KMU unter Berücksichtigung ihrer Bedingungen und Grenzen wirksamer gestaltet werden?

- Welche spezifischen Erfordernisse sind bei der Beherrschung des Innovationsprozesses in den KMU in den neuen Bundesländern zu berücksichtigen und welche Konsequenzen ergeben sich daraus?

- Inwieweit sind bewährte Ansätze des Innovationsmanagements unter marktwirtschaftlichen Bedingungen überhaupt auf die neuen Bundesländer übertragbar? Wenn ja, mit welchen Modifikationen?

- Welche Ansatzpunkte für die Weiterentwicklung des Innovationsmanagements ergeben sich aus den Umstrukturierungsprozessen in den neuen Bundesländern - einerseits für das Innovationsmanagement generell, andererseits für ähnliche Umstrukturierungsprozesse in den osteuropäischen Ländern?

- Wie ist Beratung für das Innovationsmangement in KMU generell und speziell in den neuen Bundesländern zu gestalten?

Der Technologietransfer gewinnt in diesem Zusammenhang immer mehr an Bedeutung, weil er das Innovationspotential des Unternehmens durch den Zugriff auf externes Know-how ergänzt und in vielen Fällen diesen Zugriff überhaupt ermöglicht. Technologietransfer wird allgemein als Übertragung von Wissen über neue Produkte und Verfahren - und deren Nutzung und/oder Markteinführung - von Technologie-Anbietern (-Produzenten) ggf. über Technologie-Mittler (sogenannte Agenturen) an Technologie-Anwender (-Nutzer) mit dem Ziel der Steigerung der Wettbewerbsfähigkeit der Anwender verstanden ${ }^{2}$. Er beinhaltet auch die erforderlichen organisatorischen, personellen, materiellen, informellen und finanziellen Maßnahmen, die notwendig sind, um spezifische Kenntnisse und Erfahrungen aus dem Innovationsprozeß den einzelnen Unternehmen zugänglich zu machen. Ziel ist immer die beschleunigte wirtschaftliche Nutzung von Innovationen zur Sicherung der Wettbewerbsfähigkeit der Industrie (vor allem der mittelständischen) und Stärkung ihrer Leistungsfähigkeit.

Ohne eigene Forschungs- und Entwicklungskapzitäten müssen sich gerade kleine Unternehmen technologisches Wissen aus externen Quellen beschaffen. Sie sind daher auf eine enge Kooperation mit Informationsvermittlern, Technologie-Transfer-Stellen und Forschungseinrichtungen angewiesen.

\section{Vielfältige Unterstützungsmaßnahmen seit 1990/1991}

Die Bundesregierung hat von Anfang an mit erheblichem Aufwand und über verschiedene Programme, darunter Marktvorbereitende Industrieforschung, Innovations- 
förderprogramm, PFO u. a., die Umstrukturierung der Wirtschaft und der industrienahen Forschungslandschaft unterstuitzt. Der Schwerpunkt dieser Unterstuitzung lag bei der Entwicklung neuer Technologien und deren schneller Umsetzung in Produkt- und Verfahrensinnovationen - als Schlüsselfaktor für die Wettbewerbsfähigkeit mittelständischer Unternehmen.

Parallel dazu wurde seit 1991 eine leistungsfähige und flächendeckende Technologietransfer-Infrastruktur geschaffen, vor allem durch die regional wirksam werdenden 21 Agenturen für Technologietransfer und Innovationsförderung (ATI) sowie 14 technologiespezifischen und branchenorientierten Transferzentren (TTZ).

Die Aufgabe dieser Transfereinrichtungen besteht in der Unterstützung der neu entstandenen kleinen und mittleren Unternehmen in den $\mathrm{nBL}$ in allen betrieblichen, vor allem technologischen Fragen. In der Aufbauphase lag der inhaltliche Schwerpunkt ihrer Tätigkeit häufig in der Sicherung der Überlebensfähigkeit von Unternehmen. Bei der Initiierung und Begleitung von Forschungs- und Entwicklungsprojekten sowie bei der gezielten Umsetzung von FuE-Ergebnissen in kleine und mittlere Unternehmen sind teilweise beachtliche Erfolge erreicht worden.

Trotz ihres kurzen Bestehens sind die Agenturen und die Transferstellen bekannte und gefragte Institutionen geworden. Hunderte von Unternehmen und Existenzgründern haben ihren Rat gesucht. Durch ihre Arbeit leisten sie einen wesentlichen Beitrag zum Aufbau einer wettbewerbsfähigen Industriestruktur in den neuen Bundesländern.

Dennoch zeigt sich eine Reihe von Problemen in den $\mathrm{KMU}$, die in Zukunft stärkere Beachtung finden müssen.

\section{Stärkere Konzentration auf die Schwierigkeiten und Probleme des Transfers in den KMU notwendig}

Häufig erreicht der Transfer nicht in genügendem Umfang jene Unternehmen, die seiner am dringendsten bedürfen. Großunternehmen und größere Mittelständler verfügen in der Regel über eigenes Forschungs- und Entwicklungspotential; sie sind auch in der Lage, autonom Kontakte zu Hochschulen und außeruniversitären Forschungseinrichtungen aufzubauen. Kleinunternehmen und kleinere Mittelständler verfuigen uiber derartige Kapazitäten und Kontakte in der Regel nicht; es muß daher in Zukunft verstärkt Aufgabe des Technologietransfers sein, diese Unternehmen gezielt auf Kooperationsmöglichkeiten aufmerksam zu machen und ihnen die geeigneten Partner aus dem Wissenschaftsbereich zu vermitteln.

Die Erfahrungen der ATI's und TTZ zeigen, daß trotz zahlreicher Kontakte zwischen KMU und Technologiegebern (Hochschulen, Forschungsinstitute, u. a.) erhebliche Barrieren existieren, die den direkten Transfer zwischen Technologiegebern und -nehmern beeinträchtigen bzw. teilweise unmöglich machen.
Die genannten Transferbarrieren führen dazu, daß vor allem der direkte Technologietransfer aus den Forschungsbereichen in KMU nur schwierig zustande kommt.

Wichtige Transferhindernisse in dieser Hinsicht sind ${ }^{3}$ :

\begin{tabular}{|c|c|}
\hline \multicolumn{2}{|c|}{ Transferhindernisse } \\
\hline$k$ & $\searrow$ \\
\hline Geberseite & Nehmerseite KMU \\
\hline $\begin{array}{l}\text { - nicht ausreichender Praxisbe- } \\
\text { zug: Es handelt sich in der For- } \\
\text { schung häufig um breitbandi- } \\
\text { ges Erfahrungswissen, das für } \\
\text { KMU nicht unmittelbar um- } \\
\text { setzbar ist. } \\
\text { - Angebot nicht bedarfs- (nut- } \\
\text { zer-)gerecht, relativ selten in- } \\
\text { dustriell verwertbare Techni- } \\
\text { ken (fehlende Kompatibilität) } \\
\text { - Probleme der Kommunikation } \\
\text { mit Unternehmen } \\
\text { - unterschiedliche Zeithorizonte } \\
\text { und Zielsetzungen von Wirt- } \\
\text { schaft und Wissenschaft }\end{array}$ & $\begin{array}{l}\text { - ungenügende Eigenkapitalaus- } \\
\text { stattung der KMU } \\
\text { - fehlendes qualifiziertes } \\
\text { Personal } \\
\text { - mangelhafte Einstellung ge- } \\
\text { genüber neuen Technologien } \\
\text { - Potentiale der Forschung aus- } \\
\text { zuschöpfen erfordert aufwen- } \\
\text { dige Such-, Selektions- und Auf- } \\
\text { bereitungsarbeiten } \\
\text { - unscharfe Problemdefinition } \\
\text { - Mangel bei der Beurteilung der } \\
\text { Unternehmenssituation } \\
\text { - geringer Informationsstand } \\
\text { über Transfermöglichkeiten } \\
\text { und -angebote }\end{array}$ \\
\hline
\end{tabular}

Unter Berücksichtigung des erreichten Standes sowie der kurzskizzierten Probleme und Schwierigkeiten ergibt sich eine Reihe von Anforderungen, die in Zukunft stärker beachtet werden müssen.

\section{Technologietransfer 2000 - Anforderun- gen und Vorschläge}

\subsection{Elemente des Technologietransfers im Wandel ${ }^{4}$}

Es gibt auf dem Gebiet des Technologietransfers nicht den zeitlich unbegrenzten „one best way“, wohl aber sind ausgehend von den Erfordernissen und Problemen der Unternehmen, der vorhandenen Rahmenbedingungen und der wirtschaftlichen Gesamtsituation effektive Lösungen nötig. In der wirtschaftspolitischen Praxis muß deshalb stets im Blick behalten werden, daß der Technologietransfer seine volle Wirksamkeit nur dann entfalten kann, wenn er eingebettet ist in ein umfassendes wirtschaftspolitisches Konzept, das sicherstellt, daß sich eine gesamtwirtschaftlich erwünschte Entwicklung vollzieht.

Unter Berücksichtigung des erreichten Standes und der zukünftigen Anforderungen ist ein Umdenken im Technologietransfer anzustreben. Die bisher überwiegende Angebotsorientierung bestand darin, daß sich die Transfereinrichtungen bemüht haben, in Hochschulen, Forschungseinrichtungen und Unternehmen gewonnene Erkenntnisse und entwickelte Produkt- und Verfahrensideen nach fertiger Entwicklung an die Industrie heranzutragen. Eine Nachfrageorientierung muß systematisch den Problemlösungsbedarf in den betroffenen Unternehmen erfassen, daraufhin die geeigneten Kooperationspartner vermitteln und ggf. in den Forschungsbereich hinein Entwicklungsanstöße geben, die gezielt auf den Problemlösungsbedarf der Unternehmen gerichtet sind. 
Von den zwei Formen des Technologietransfers, die sich grundsätzlich herausgebildet haben, angebotsorientierter Transfer (technology push) und nachfrageorientierter Transfer (market pull) gewinnt richtigerweise letztere an Gewicht. Hinzu kommt eine Komponente, die für die Umsetzung der Technologietransferkonzepte zunehmend an Bedeutung gewinnt, und zwar die Ausbildung und intensivere Nutzung von Netzwerken. Analysen der FuE-Strategien technologieintensiver Firmen zeigen, daß Netzwerke und Kooperationen zu einem immer wichtigeren Bestandteil des Technologietransfers werden. Innovationen sind offenbar immer weniger Ergebnis isolierter Aktivitäten in Unternehmen, sondern werden zunehmend in bi- und multilateralen Beziehungen und Kooperationen entwickelt, und zwar sowohl im nationalen wie im internationalen Rahmen. Die wachsende Differenziertheit/Arbeitsteilung der wirtschaftlichen Tätigkeit sowie von Forschung und Wissenschaft erfordert eine stärker vernetzte Kommunikations- und Computer-Netz-Infrastruktur: Sie muß unterschiedliche autonome und integrierte Verbünde (Forschung, Produktion, Marketing, Management, Unternehmen, Region, Land, internationale Einrichtungen) ermöglichen. Das schließt auch die gezielte Nutzung von elektronischen Netzen (z. B. Internet) ein.

Mit diesem Herangehen an den Technologietransfer und die Innovationsförderung bilden sich immer stärker Elemente eines Technologietransfers einer neuen Generation heraus.

Zu enge Unternehmensstrategien allein durch primär technisch orientiere Innovationsförderung zu verbessern, wird den heutigen Bedingungen nicht mehr gerecht. Immer stärker geht es darum, auf Grundlage ganzheitlicher Betrachtung durch Technologietransfer und Innovationsförderung die unternehmerische Tätigkeit unter wirtschaftlichen Kriterien aus der Sicht des Kunden zu optimieren. Letztlich ist dies mit einer Neudefinition unternehmerischen Handelns verbunden.

\section{Zukünftige Anforderungen}

\section{a) Ausrichtung des Technologietransfers auf Wachs- tumstechnologien - stärker zu erreichende Effekte}

Es zeichnen sich (entsprechend durchgeführter Untersuchungen und absehbarer nationaler und internationaler Trends) langfristige Entwicklungsschwerpunkte (Wachstumstechnologien) ab, auf die innovative Unternehmen und staatliche Fördermaßnahmen auf dem Gebiet des Technologietransfers besonders orientiert bzw. konzentriert werden müssen ${ }^{5}$. Das Ganze muß zugleich mit Blick auf die EU und auf mögliche Nachfrageentwicklungen aus Mittel- und Osteuropa betrachtet werden. Solche Gebiete sind z. B:

- Mikrosystemtechnik/Mikroelektronik,

- Optoelektronik,

- Informations- und Kommunikationstechnologien/ Fuzzy- und Neuro-Technologien (Telematik, Computer, Software, Netzwerke, Sprachen- und Bildverarbeitung),

- Produktionsautomatisierung - Sensoren,

- Biotechnologie.
Von besonderer Bedeutung für das konzeptionell-methodische Vorgehen sind dabei jene Effekte, die mit dem Technologietransfer erreicht werden sollen. Hier sind besonders zu nennen:

- die Neu- und Weiterentwicklung von Produkten,

- die Verbesserung oder Neueinführung von technologisch fortgeschrittenen Produktionsverfahren und anlagen,

- die Innovation bei organisatorischen Systemen und Abläufen,

- die Verbesserung des Marktzugangs und der Marktbearbeitung.

Im Vordergrund stehen hierbei die beiden zuerst genannten Anwendungen, d. h. die Innovation von Produkten oder Produktionsverfahren. Dabei zeigt die bisherige Erfahrung in den neuen Bundesländern, daß Innovation in diesem Bereich sehr häufig mit Innovationen auch bei organisatorischen oder marktbezogenen Fragestellungen verknüpft sind.

b) Technologietransfer in seiner Vielfalt entwickeln In stärkerem Maße ist darauf abzuzielen, den Technologietransfer in seiner Vielfalt zu entwickeln und auszubauen. Das betrifft vor allem folgende Transferbeziehungen ${ }^{6}$ :

- Forschung $\Rightarrow$ Unternehmen

Diese Zusammenarbeit von universitären oder außeruniversitären Forschungseinrichtungen mit Unternehmen zwecks gemeinsamer Entwicklung einer Produkt- oder Verfahrensinnovation kennzeichnet den Technologietransfer im eigentlichen Sinne.

- Unternehmen $\Rightarrow$ Unternehmen

Dieser als „Diffusion“ umschriebene Vorgang der Verbreitung technologischer Neuerungen zwischen verschiedenen Unternehmen kann unterschiedliche Ausprägungen annehmen. Besondere Bedeutung kommt hierbei den Transferbeziehungen zwischen Großunternehmen und KMU bei.

Die Entwicklung und Durchsetzung von Modellen des Technologietransfers, die durch die Kombination von Technologienachfrage und -angebote charakterisiert sind, ist stärker anzustreben. Einen Fortschritt in dieser Hinsicht verspricht der in Berlin entwickelte „TransferRing“. Der TransferRing ist eine gemeinsame Initiative der Industrie- und Handelskammer zu Berlin, der Technologie-Vermittlungs-Agentur Berlin e. V. und Fachhochschulen aus Berlin und Brandenburg. Er vereint Berater und praxiserfahrene Professoren zu einem interdisziplinären Team, das Unterstuitzung für kleine und mittlere Unternehmen anbietet, sowie externe Entwicklungs- und Forschungsleistungen bereitstellt. Bisher wurden bereits über 70 Projekte in den Bereichen Verkehrstechnik, Werkstofforschung, Holzverarbeitungsmaschinen und -anlagen sowie Medizintechnik erfolgreich bearbeitet.

Der TransferRing koppelt den nachfrageorientierten Technologietransfer mit systematischen angebotsorientierten Komponenten. Ein technologietransferbegleitendes Projektmanagement hilft den Unternehmen, sich 
auf neue Technologien einzustellen und die Projekte rationell zu bearbeiten.

\section{c) Personenorientierter Technologietransfer}

Die Bedeutung des „Transfers über Köpfe“ ist allgemein bekannt. Hier sollte künftig mehr über unkonventionelle Ansätze nachgedacht werden. Die momentane Einbahnstraße des Personaltransfers aus der Forschung in die Praxis muß durch neue Modelle ergänzt werden. Überlegt werden könnte z. B., Unternehmensmitarbeiter für 2 Jahre an Hochschulen zu entsenden oder ihnen ein mehrfaches Pendeln zwischen Forschung und Wirtschaft zu ermöglichen.

Eine andere, insbesondere für größere Unternehmen gangbare Variante stellt die Bildung von sogenannten „Pilotentwicklungsgruppen“ dar, die folgende Aufgaben gemeinsam lösen sollten:

- Gemeinsame Umsetzung neuer FuE-Ergebnisse in wirtschaftliche Anwendungen (Welche Produkte und Verfahren resultieren aus den FuE-Ergebnissen, wo sind die Märkte, wer kommt als Produzent in Frage usw.?);

- Gemeinsame Bearbeitung technologischer Herausforderungen mehrerer Unternehmen (Definition der Aufgabe, Prüfung hinsichtlich der Frage, wer die Aufgabe lösen kann und ob ggf. eine Förderung greift);

- Optimierung der Wirkungskette zwischen Technologie-Entwicklung, Technologie-Produzent und Technologie-Anwender;

- Organisation von Verbundprojekten;

- Erörterung von Technologie-Trends/Markt-Trends in den einzelnen Technologie-Clustern.

Solche Arbeitskreise und -gruppen müssen in stärkerem Maße initiiert und finanziell unterstiitzt werden.

\section{Existenzgründungen}

Eng verbunden mit dem personenorientierten Technologietransfer ist das Gründungsgeschehen speziell aus Wissenschaftseinrichtungen, zur direkten wirtschaftlichen Umsetzung neu entstandenen Wissens.

Der große Rückstand bei Existenzgründungen industrieller KMU gegenüber den alten Bundesländern erfordert größere Anstrengungen zur Mobilisierung regionaler Gründungspotentiale (regelmäßige Innovations- und Gründungswochen, Motivationskampagne Fachhochschulen, Großunternehmen-Sponsoring für Existenzgründungen u. a.). Dies sollte aus politischer Sicht besonders unterstuitzt werden.

Als aussichtsreiche Variante im Gründungsgeschehen wird die Bildung gemeinsamer Gesellschaften von Unternehmen und Forschungseinrichtungen angesehen, die unter einheitlicher Managementverantwortung stehen. Diese Möglichkeit wird bisher kaum genutzt. Ursachen hierfür sind administrative Hemmnisse und die Schwierigkeit, praxisorientierte, interdisziplinär arbeitende Teams zusammenzubringen.

Die Unterstützung der Gründung innovativer kleiner und mittlerer Unternehmen, die die Wirtschaftsstruk- tur gewissermaßen „von unten“ erneuern, sollte ebenfalls durch die Gewährung von Steuervergünstigungen, Krediterleichterungen, gezielten Fördermaßnahmen und unbürokratischen Genehmigungsverfahren, vor allem unter Reintegration des brachliegenden endogenen Forschungspotenials besonders gefördert werden.

Unterstützt wird der Vorschlag des ifo-Instituts München, durch die öffentliche Hand einen Venture-CapitalFonds fuir junge wachstumsintensive High-Tech-Unternehmen bereitzustellen ${ }^{7}$.

\section{d) Engere Verflechtung von Grundlagenforschung, angewandter industrienaher Forschung sowie in- novativer Produkte und Leistungen 8}

Dies erweist sich als dringend notwendig, um den neuen Herausforderungen der technologischen Entwicklung, der veränderten Dynamik des Weltmarktes, des wachsenden Erneuerungsbedarfs der Industrie gewachsen zu sein. Die Wirtschaftspolitik kann hierzu durch eine stärkere Konzentration und Orientierung auf industrienahe Forschungsaktivitäten, die für die Entwicklung neuer marktfähiger Produkte und Verfahren besonders bedeutsam sind, wirksam beitragen.

Aktuelle Untersuchungen (z. B. der Forschungsagentur Berlin) unterstreichen, daß nur ein geringer Teil der an den Universitäten und Hochschulen durchgeführten Grundlagenforschung in eine anwendungsorientierte Forschung mündet. Es sollten die Anreize für Technologieanbieter erhöht werden, ihr Wissen direkt an die Technologienachfrager zu verkaufen. Auch die Praxis der Projektförderung trägt nicht dazu bei, Industrienähe zu befördern. Projekte, die weit entfernt sind von Produkt und Technologie, haben häufig bessere Chancen als Projekte, die sich in der Nähe der industriellen Realisierung bewegen. (Eine Ausnahme bildet das Programm „Marktvorbereitende Industrieforschung“ des BMWi).

Die Industrie wird künftig in stärkerem Maße aus dem Forschungsbereich neue wissenschaftliche Ideen, die sich aus einem breiten Wissenschaftsspektrum herleiten, sowie Prinziplösungen „abverlangen“ und vermehrt eine verbesserte Anwendungsreife neuer Lösungen erwarten. Das setzt Akzente in der Zusammenarbeit von Forschung und KMU und modifiziert bisher angewandte Formen für den Erkenntnistransfer aus dem Forschungsbereich in die Wirtschaft. Wissenschaftler und Forscher werden in Zukunft weitaus stärker herausgefordert werden, neue Erkenntnisse zu vermitteln und praxisnahe Innovationen anzuregen. Unter Beachtung dieser Gesichtspunkte gilt es, in den nächsten Jahren die gegenseitige Verankerung von Fachleuten aus Wirtschaft und Wissenschaft in Gremien, Arbeitskreisen und -gruppen auszubauen, langfristige Forschungsaufgaben, die auf Erkenntnisfortschritt und Innovation zielen, nach einer einheitlichen Konzeption zu bearbeiten, zusätzliche Möglichkeiten für die Arbeit gemeinsamer Forschungsteams zu schaffen, die Zusammenarbeit von Wissenschaftlern mit den Unternehmen zu unterstützen sowie im Gerätebau und in der Bereitstellung/Beschaffung von Forschungstechnik gemeinsame Wege zu gehen. 


\section{Anmerkungen}

1 Vgl. Ansoff, H.I. (1966; 1981 a; 1981 b); Gälweiler, A. (1974, 1990); May, A./Maijluf, N. (1988); Hinterhuber, H.H. (1992); Mann, R. (1988); Probst, G./Gomez, P. (1989); Ulrich, H./ Probst, G. (1988); Fritsch, M. (1985), S. 4

2 Vgl. Böhler, H. (1998)

3 Vgl. Geschka, H. (1990), S. 30; Staudt, E. (1990), S. 205-206

4 Vgl. ifo Institut (1995), S. 55-58; ESPRIT Club NRW (1994), S. 5-6; Corsten, H. (1982), S. 210, S. 287

5 Vgl. „Leitprojekte des BMBF“, die in dieser Hinsicht wichtige Orientierungen und Hinweise enthalten.

6 Vgl. PROGNOS: (1991), S. $4 \mathrm{ff}$.

7 Vgl. Reinhard, M./Schmalholz, H., (1996)

8 Vgl.: Brockhoff, K. (1964), S. 20, 21, 29; Wolfrum, B. (1991), S. 8-10; Thom, N. (1980), S. 35; Schuster, H.-W. (1985)

\section{Literatur}

- Ansoff, H.I. (1966: Corporate strategy, New York 1966 dtsch.: Management Strategien, München 1966

- Ansoff, H.I. (1981 a): Strategic Management, London 1981

- Ansoff, H.I. (1981 b): Die Bewältigung von Überraschungen und diskontinuitäten durch Unternehmensführung - Strategische Reaktion auf schwache Signale. In: Steinmann, H. (Hrsg.): Planung und Kontrolle, München 1981

- Böhler, H. u. a.: Der Technologie-Transfer in einer strukturschwachen Region, Universität Bayreuth 1988

- Brockhoff, K. (1994): Forschung und Entwicklung: Planung und Kontrolle, München/Wien 1994

- Corsten, H. (1982): Der nationale Technologietransfer, Formen - Elemente - Gestaltungsmöglichkeiten - Probleme, Berlin 1982

- ESPRIT CLUB NRW (1994): Technologietransfer für mittelständische Unternehmen in Nordrhein-Westfalen - Ergebnisse einer Befragung der Mitglieder des ESPRIT Clubs NRW, 1994

- Fritsch, M. (1985): Führungskräftefortbildung bei innovationsorientierter Unternehmensführung, Frankfurt am Main/Bern/New York 1985

- Gälweiler, A. (1974): Unternehmensplanung Grundlagen und Praxis, Frankfurt am Main 1974

- Gälweiler, A. (1990): Strategische Unternehmensführung, Frankfurt/Main 1990

- Geschka, H. (1990): Erkenntnisse der Innovationsforschung - Konsequenzen für die Praxis. In: Neue Produkte: Anstöße, Wege, realisierte Strategien, VDI-Berichte 724, Düsseldorf 1990

- Hax, A./Majluf, N. (1988): Strategisches Management: Ein integratives Konzept aus dem M.I.T., Frankfurt/New York 1988

- Hinterhuber, H.H. (1992): Strategische Unternehmensführung, Bd. $1+2$, Berlin, New York 1992

- Ifo Institut (1995): Der Beitrag des Technologietransfers zur Steigerung der Wettbewerbsfähigkeit der deutschen Wirtschaft - Stand und Reformbedarf, Gutachten des ifo Instituts für Wirtschaftsforschung im Auftrag des Bundesministeriums für Wirtschaft, München 1995

- Mann, R. (1988): Das ganzheitliche Unternehmen, München 1988

- Probst, G./Gomenz, P. (1989): Die Methodik vernetzten Denkens zur Lösung komplexer Probleme. In: Probst, G./ Gomenz, P. (Hrsg.): Vernetztes Denken - Ganzheitliches Führen in der Praxis, S. 3 - 20, Wiesbaden 1989, 2. Auflage 1991
- PROGNOS (1991): Rothkirch, Chr. von/Wolff, H.: Möglichkeiten zur Verbesserung des Technologietransfers in den Ländern der ehemaligen DDR, Kurzuntersuchung im Auftrag des BMWi, Köln/Basel 1991

- Reinhard, M./Schmalholz, H.: Technologietransfer in Deutschland - Stand und Reformbedarf, 1996 Dunker \& Humblot Berlin

- Schuster, H.-W. (1985): Innovationsberatung für mittlere Industrieunternehmen, Forschungsberichte VDI, Reihe 16 : Technik und Wirtschaft, Nr. 29, Düsseldorf 1985

- Staudt, E. (1990): Die betriebswirtschaftliche Bedeutung von Innovationen. In: Schuster, H.J. (Hrsg.): Handbuch des Wissenschaftstransfers, Berlin/Heidelberg/New York/... 1990

- Thom, N. (1980): Grundlagen des betrieblichen Innovationsmanagements, Königsstein/Ts. 1980

- Ulrich, H./Probst, G. (1988): Anleitung zum ganzheitlichen Denken und Handeln - ein Brevier für Führungskräfte, Bern/Stuttgart 1988

- Wolfrum, B. (1991): Strategisches Technologiemanagement, Wiesbaden

\section{Verfasser}

\section{Prof. Dr. Klaus Däumichen}

Geschäftsführer des Verbandes der Innovations- und Technologieberatung-Organisationen Deutschlands e.V. (VITO e.V.)

Stellv. Vorsitzender des Technologieforums Adlershof; TSB Technologiestiftung Innovationsagentur Berlin GmbH

Leiter der Geschäftsstelle Adlershof Rudower Chaussee 5, IGZ, 12489 Berlin Tel. (0 30) 63925171 